\title{
Consenso | Protocolo Brasileiro para Infecções Sexualmente Transmissíveis 2020: infecções que causam corrimento uretral
}

doi: 10.1590/S1679-4974202100009.esp1

\author{
Brazilian Protocol for Sexually Transmitted Infections 2020: infections that \\ cause urethral discharge \\ Protocolo Brasileño para Infecciones de Transmisión Sexual 2020: infecciones \\ que causan secreción uretral
}

\author{
Leonor Henriette de Lannoy' - (1) orcid.org/0000-0002-9520-8538 \\ Roberto José de Carvalho da Silva² - (1) orcid.org/0000-0001-9186-0206 \\ Edilbert Pellegrini Nahn Júnior ${ }^{3}$ - (1) orcid.org/0000-0003-1656-3441 \\ Eduardo Campos de Oliveira4 - (1) orcid.org/0000-0001-7066-9904 \\ Pâmela Cristina Gaspar ${ }^{1}$ - (1) orcid.org/0000-0003-4642-0783 \\ 'Ministério da Saúde, Secretaria de Vigilância em Saúde, Brasília, DF, Brasil \\ ${ }^{2}$ Secretaria de Estado da Saúde de São Paulo, Programa Estadual de DST/Aids, São Paulo, SP, Brasil \\ ${ }^{3}$ Universidade Federal do Estado do Rio de Janeiro, Campus Macaé, RJ, Brasil \\ ${ }^{4}$ Secretaria de Estado da Saúde de Santa Catarina, Gerência Estadual de IST/Aids/HV, Florianópolis, SC, Brasil
}

\section{Resumo}

Este artigo aborda as infecções que causam corrimento uretral, tema que compõe o Protocolo Clínico e Diretrizes Terapêuticas para Atenção Integral às Pessoas com Infecções Sexualmente Transmissíveis, publicado pelo Ministério da Saúde do Brasil em 2020. Tal documento foi elaborado com base em evidências científicas e validado em discussões com especialistas. As uretrites, quando não tratadas de maneira correta, ou quando o microrganismo desenvolve resistência ao tratamento empregado, podem causar danos graves e até irreversíveis à saúde. Os níveis de resistência antimicrobiana que esses agentes têm desenvolvido são considerados uma emergência global em saúde pública. Neste artigo, são apresentados aspectos epidemiológicos e clínicos, recomendações sobre diagnóstico e tratamento e estratégias para as ações de vigilância, prevenção e controle das infecções que causam corrimento uretral, com a finalidade de contribuir com gestores e profissionais de saúde para a qualificação da assistência.

Palavras-chave: Uretrite; Neisseria gonorrhoeae; Chlamydia trachomatis; Protocolos Clínicos; Saúde Pública.

Endereço para correspondência:

Leonor Henriette de Lannoy - Ministério da Saúde, Secretaria de Vigilância em Saúde, SRTVN Quadra 701, Via W 5 Norte, Lote D, Edifício PO 700, Brasília, DF, Brasil. CEP: 70719-040

E-mail: leonordelannoy@gmail.com 


\section{Apresentação}

0 artigo aborda as infecções que causam corrimento uretral, tema que compõe o Protocolo Clínico e Diretrizes Terapêuticas (PCDT) para Atenção Integral às Pessoas com Infecções Sexualmente Transmissíveis (IST), publicado pela Secretaria de Vigilância em Saúde, do Ministério da Saúde do Brasil. Para a elaboração do PCDT, foram realizadas a seleção e a análise das evidências disponíveis na literatura e discussão em um painel de especialistas. 0 referido PCDT foi aprovado pela Comissão Nacional de Incorporação de Tecnologias no Sistema Único de Saúde (Conitec) ${ }^{1}$ e atualizado pelo grupo de especialistas em IST em 2020.

\section{São fatores associados à uretrite idade jovem, baixo nível socioeconômico, múltiplas parcerias ou nova parceria sexual, histórico de IST, uso irregular de preservativos e falta de acesso a diagnóstico e tratamento adequados.}

\section{Aspectos epidemiológicos}

A uretrite é definida como uma inflamação da uretra, podendo ser infecciosa ou não. Vários agentes podem causar uretrite infecciosa, no contexto de práticas sexuais sem preservativos. As uretrites são classificadas, de acordo com o agente etiológico, em gonocócicas, causadas por Neisseria gonorrhoeae, e em não gonocócicas, causadas majoritariamente por Chlamydia trachomatis e Mycoplasma genitalium. Outros agentes, como Trichomonas vaginalis, enterobactérias (nas relações anais insertivas), 0 vírus do herpes simples (herpes simplex virus, HSV), o adenovírus e Candida sp. são menos frequentes. ${ }^{2,3}$

São fatores associados à uretrite: idade jovem, baixo nível socioeconômico, múltiplas parcerias ou nova parceria sexual, histórico de IST e uso irregular de preservativos, bem como falta de acesso a diagnóstico e tratamento adequados. ${ }^{2,3}$

A Organização Mundial da Saúde (OMS), por meio de estudo de revisão sistemática, estimou, para 0 ano de 2016, a ocorrência de 370,4 milhões de novas infecções urogenitais curáveis por clamídia, gonorreia, e tricomoníase em mulheres e homens de 15 a 49 anos. ${ }^{4}$ A taxa de incidência global de clamídia em 2016 foi de 34 casos por 1.000 mulheres e 33 casos por 1.000 homens; de gonorreia, 20 casos por 1.000 mulheres e 26 casos por 1.000 homens; e, de tricomoníase, 40 casos por 1.000 mulheres e 42 casos por 1.000 homens. ${ }^{4}$ Estudo de revisão sistemática e metanálise sobre a prevalência de $M$. genitalium, que contemplou três estudos com um total de 3.809 pessoas, estimou uma prevalência de 1,3\% desse agente em países desenvolvidos e 3,9\% em países em desenvolvimento. A prevalência foi similar em homens e mulheres. ${ }^{5}$ Análises do sistema europeu de vigilância de doenças transmissíveis verificaram que, em 2018, dos 17 países que coletaram dados sobre 0 modo de transmissão de gonorreia, $48 \%$ de todos os casos confirmados e notificados ocorreram em homens que fazem sexo com homens. ${ }^{6}$

No Brasil, o cenário epidemiológico das infecções que causam corrimento uretral acompanha os altos índices mundiais. Estima-se que a prevalência de gonorreia na população de 15 a 49 anos seja de aproximadamente $1,4 \%$ e que a incidência na população geral esteja em torno de 500.000 novos casos por ano. ${ }^{7}$

Quanto ao risco de transmissão dos agentes infecciosos, verifica-se que a $N$. gonorrhoeae é transmitida do homem infectado para a mulher, com probabilidade que pode variar de $50 \%$ a $73 \%$, independentemente do número de exposições. Já a probabilidade de o homem se infectar com mulher infectada varia de $20 \%$ a $35 \%$ em uma exposição. ${ }^{8} 0$ sexo oral desprotegido resulta em infecção em cerca de $25 \%$ das vezes, pois a faringe é um dos maiores reservatórios assintomáticos do patógeno. ${ }^{9}$

No caso da $C$. trachomatis, a probabilidade de um homem contrair a infecção a partir de uma mulher é de 32\%, e de a mulher contrair a infecção do homem, de 40\%; essa proporção de transmissão se refere a contato com parceiro infectado por ato sexual desprotegido. ${ }^{10}$ Embora esteja bem estabelecido que $0 \mathrm{M}$. genitalium é sexualmente transmitido, não se sabe quantas vezes isso ocorre por episódio de relação sexual desprotegida. ${ }^{11}$ Estudos sugerem que a transmissão de $M$. genitalium é provavelmente menor do que a de $C$. trachomatis, o que seria consistente com a carga infecciosa desses agentes. É provável que homens com uretrite não gonocócica sintomática e, presumivelmente, cargas mais altas de 


\begin{tabular}{|l|c|c|c|c|}
\hline Agente infeccioso & Prática sexual & $\begin{array}{c}\text { Transmissão do homem } \\
\text { para mulher }\end{array}$ & $\begin{array}{c}\text { Transmissão da mulher } \\
\text { para o homem }\end{array}$ & Referência \\
\hline \multirow{2}{*}{ Neisseria gonorrhoeae } & Relação sexual & $50 \%$ a $73 \%$ & $20 \%$ a 35\% & {$[8]$} \\
\cline { 2 - 5 } & Sexo oral & $25 \%$ & $25 \%$ & {$[9]$} \\
\hline Chlamydia trachomatis & Relação sexual & $40 \%$ & $32 \%$ & {$[10]$} \\
\hline Trichomonas vaginalis & Relação sexual & Não há informação & $22 \%$ a 72\% & {$[14]$} \\
\hline
\end{tabular}

Figura 1 - Risco de transmissão dos agentes infecciosos causadores de corrimento uretral em práticas sexuais desprotegidas

M. genitalium sejam mais infecciosos do que homens com infecção assintomática. ${ }^{12,13}$

0 risco de os homens se infectarem com parceiras infectadas por T. Vaginalis varia de $22 \%$ a $72 \%$. Tendo em vista que há contaminação em até $5 \%$ das pessoas que relatam sexo anal receptivo recente, é necessário esclarecer se o reto pode ser um reservatório para infecção por T. Vaginalis. ${ }^{14}$ De acordo com o Centro de Prevenção e Controle de Doenças dos Estados Unidos (Centers for Disease Control and Prevention, CDC) não são recomendados testes retais e orais para T. Vaginalis devido à falta de evidências dessas infecções nesses locais.

A Figura 1 apresenta síntese dos riscos oferecidos por cada agente infeccioso causador de corrimento uretral.

\section{Aspectos clínicos}

0 corrimento uretral é uma síndrome clínica, com identificação de um grupo de sintomas e sinais comuns a determinadas infecções, caracterizada por corrimento de aspecto que varia de mucoide a purulento, com volume variável, podendo estar associado a dor uretral (independentemente da micção), disúria, estrangúria (micção lenta e dolorosa), prurido uretral e eritema do meato uretral. 0 aspecto do corrimento uretral purulento corresponde a $75 \%$ das uretrites causadas por $N$. gonorrboeae e de $11 \%$ a $33 \%$ das uretrites não gonocócicas; por sua vez, o aspecto mucoide apresenta-se em cerca de $25 \%$ dos casos de uretrites gonocócicas e 50\% dos casos de uretrites não gonocócicas. ${ }^{2}$

A uretrite gonocócica é um processo infeccioso e inflamatório da mucosa uretral. Após o período de incubação, que dura em média de dois a cinco dias, variando de um a 10 dias, a infecção evolui sintomaticamente. A disúria é compreendida como a sensação de formigamento e prurido intrauretral seguido de dor à micção. 0 corrimento, inicialmente mucoide, torna-se purulento em um a dois dias, com grande volume e acompanhado de edema do meato uretral. Alguns homens podem apresentar febre, assim como manifestações de uma infecção aguda sistêmica. Cerca de $95 \%$ dos casos tornam-se assintomáticos em três meses, e uma proporção de casos não tratados pode evoluir para cura espontânea em algumas semanas. ${ }^{15}$

As complicações clínicas pelo não tratamento imediato ocorrem em até $50 \%$ dos casos, pela progressão da infecção para a uretra posterior. A epididimite aguda compreende a complicação mais frequente, podendo evoluir com obstrução do ducto epididimário, determinando oligospermia, azoospermia e esterilidade. Outras consequências da uretrite gonocócica são: prostatite, orquite, edema peniano (em particular do prepúcio), balanopostite, e, ainda, lesões das glândulas sebáceas e acinosas da região genital, tisonites (glândulas de Tyson), cowperites (glândulas de Cowper) e litrites (glândulas de Littré). ${ }^{15}$

A uretrite não gonocócica é a uretrite sintomática cuja bacterioscopia pela coloração de Gram, cultura e detecção de material genético por biologia molecular são negativas para o gonococo. A infecção por clamídia no homem é responsável por aproximadamente 50\% dos casos de uretrites não gonocócicas. 0 período de incubação no homem é de 14 a 21 dias. Estima-se que dois terços das parceiras estáveis de homens com uretrite não gonocócica hospedem a $C$. trachomatis na endocérvice, podendo, assim, reinfectar seus parceiros sexuais e desenvolver quadro de doença inflamatória pélvica se não for realizado tratamento. ${ }^{16-18}$

No homem, a uretrite não gonocócica caracteriza-se, habitualmente, pela presença de corrimentos mucoides, discretos, com disúria leve e intermitente. 


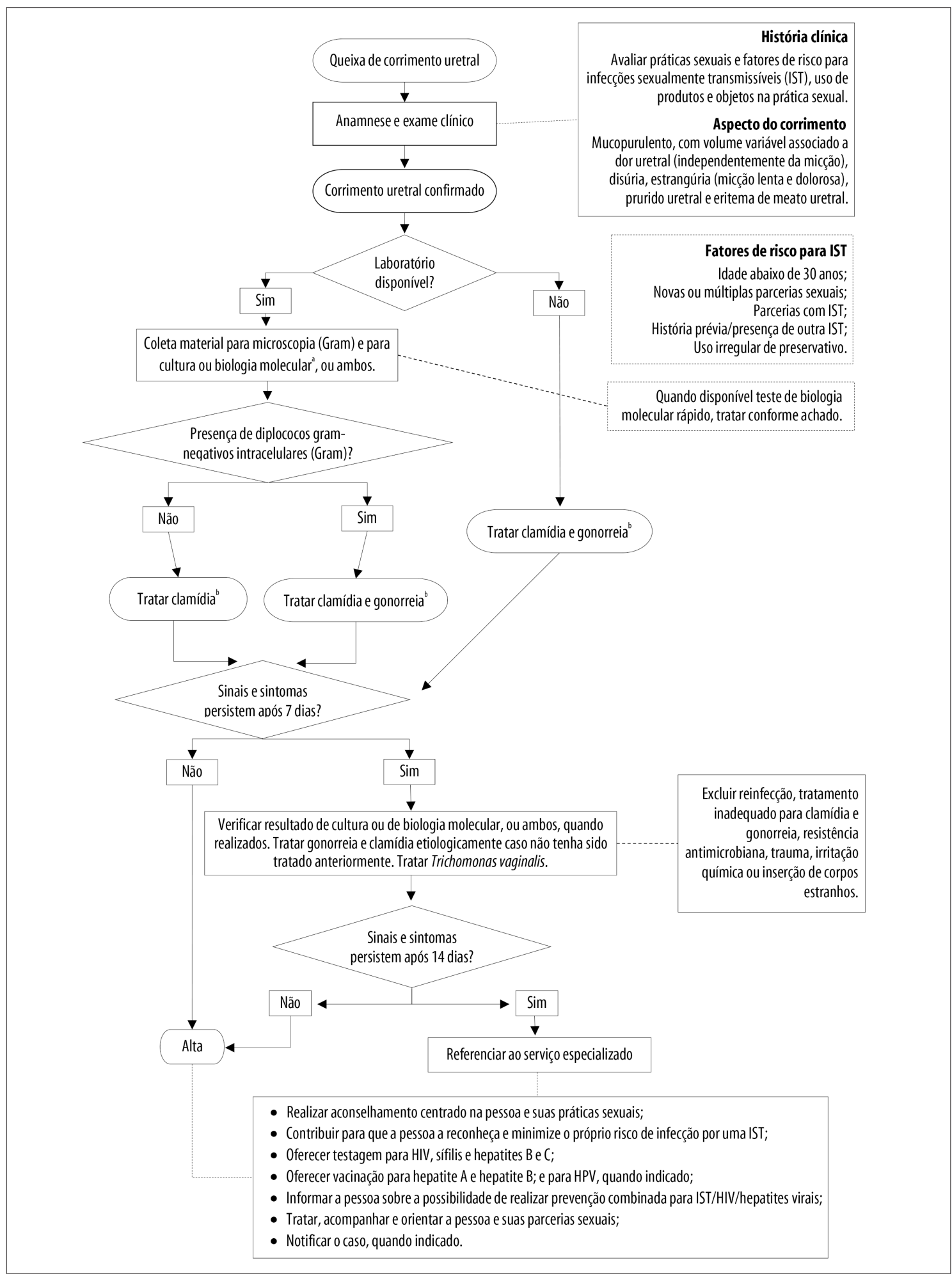

Fonte: adaptado do Protocolo Clínico e Diretrizes Terapêuticas para Atenção Integral às Pessoas com Infecções Sexualmente Transmissíveis, $2020 .{ }^{2}$

Notas: a) Existem kits de biologia molecular que detectam mais patógenos simultaneamente, além de clamídia e gonococo, e que também são úteis para o diagnóstico etiológico de uretrites, como M. genitalium; b) Esquema terapêutico também contempla tratamento para M. genitalium.

\section{Figura 2 - Recomendações para atendimento às pessoas com queixa de corrimento uretral}


A uretrite subaguda é a forma de apresentação em aproximadamente $50 \%$ dos homens com uretrite causada por $C$. trachomatis. ${ }^{19}$ Entretanto, em alguns casos, os corrimentos das uretrites não gonocócicas podem simular, clinicamente, os da gonorreia. As uretrites causadas por $C$. trachomatis podem evoluir para prostatite, epididimite, balanite, conjuntivite (por autoinoculação) e síndrome uretro-conjuntivo-sinovial ou síndrome de Reiter.

As mulheres com uretrite apresentam disúria leve, que pode estar associada a corrimento vaginal ou sangramento. 0 exame físico pode revelar a presença de cervicite mucopurulenta ou até mesmo lesões herpéticas símiles vulvovaginais. ${ }^{20}$

Nos casos de uretrites persistentes, deve-se realizar a avaliação principalmente por meio da história clínica, considerando a possibilidade de reinfecção ou tratamento inadequado para clamídia e gonorreia. Descartadas tais situações, devem-se pesquisar agentes não suscetíveis ao tratamento anterior (por exemplo, M. genitalium e T. Vaginalis), bem como a ocorrência de resistência aos antimicrobianos. ${ }^{2}$

Outras causas não infecciosas de uretrites, como trauma (ordenha continuada), instrumentalização, inserção de corpos estranhos intrauretrais ou parauretrais (piercings) e irritação química (uso de produtos lubrificantes e espermicidas) devem ser consideradas no diagnóstico diferencial de uretrites persistentes. ${ }^{2}$

\section{Diagnóstico}

0 manejo do corrimento uretral pode ser realizado por meio da abordagem sindrômica para situações em que não há suporte laboratorial',21,22 (Figura 2). Para os casos de corrimento uretral masculino, estima-se que a abordagem sindrômica tenha uma sensibilidade que varia de $84 \%$ a $95 \%{ }^{23}$

A utilização de testes diagnósticos é indicada para 0 rastreamento dos casos assintomáticos de uretrite e para investigação dos casos sintomáticos. ${ }^{2} 0$ bom desempenho dos testes depende do rigoroso cumprimento de todas as etapas preconizadas pelos fabricantes, incluindo coleta, transporte e armazenamento das amostras. ${ }^{24}$

0 rastreamento dos casos assintomáticos de uretrite deve ser realizado utilizando-se técnicas de biologia molecular, que incluem métodos com elevada sensibilidade e especificidade, como a reação em cadeia da polimerase e a amplificação mediada por transcrição. Esses testes, que se baseiam em amplificação de ácidos nucleicos (nucleic acid amplification test, NAAT), permitem a utilização de amostras de corrimento uretral masculino ou urina, ou ambos os tipos de amostra, e a identificação de um ou mais patógenos simultaneamente em uma única amostra, a depender do fabricante. Os resultados são emitidos discriminando-se os patógenos detectados. ${ }^{24-27}$

Para o diagnóstico de uretrites sintomáticas, podem ser realizados diversos testes para auxiliar na identificação do agente causador. ${ }^{2}$ Estão incorporados ao Sistema Único de Saúde (SUS) brasileiro a bacterioscopia, a cultura e os métodos de biologia molecular, disponíveis de forma heterogênea nos diferentes níveis de atenção dos serviços de saúde.

$\mathrm{Na}$ uretrite gonocócica, é possível visualizar, por meio da bacterioscopia, diplococos Gram-negativos intracelulares em leucócitos polimorfonucleares. 0 método possui alta sensibilidade e especificidade em amostras de corrimento uretral masculino. ${ }^{2,24,27} \mathrm{~A}$ cultura de amostras em meio seletivo (por exemplo, Thayer-Martin ou similar) é uma técnica com boa sensibilidade e alta especificidade para diagnóstico de $N$. gonorrboeae, desde que empregada com rigor metodológico. ${ }^{27}$ Em suma, realiza-se o cultivo de amostras biológicas em meios de cultura que favoreçam 0 crescimento de Neisseria sp. Como o meio de cultura também permite o crescimento de outras espécies do gênero Neisseria, é importante que se faça o Gram das colônias típicas (visualização de diplococos Gram-negativos) e testes de oxidase e catalase, que deverão apresentar resultados positivos quando se tratar de $N$. gonorrboeae. Para diagnóstico definitivo, podem-se submeter as colônias a provas bioquímicas (manuais ou automatizadas), pois o comportamento particular das bactérias na presença de diversos substratos possibilita a identificação das espécies de Neisseria.,28,29 A cultura também permite a investigação do perfil de susceptibilidade do gonococo aos antimicrobianos por meio de técnicas para determinação da concentração inibitória mínima, e possui grande utilidade para fins de vigilância e estabelecimento de diretrizes de tratamento. ${ }^{24,29}$ Além da bacterioscopia e da cultura, estão disponíveis no SUS as técnicas de biologia molecular, como os NAAT, que apresentam ótimo desempenho para diagnóstico etiológico das uretrites sintomáticas 
e são fortemente recomendados no tratamento etiológico do corrimento uretral.

Na ausência dos métodos citados, é possível a utilização de testes que sugerem presença de infecção, porém não definem 0 agente infeccioso, como o teste positivo de esterase leucocitária na urina de primeiro jato ou o exame microscópico de sedimento urinário de primeiro jato apresentando mais de dez leucócitos polimorfonucleares por campo (aumento de 1.000 vezes) ${ }^{2}$

\section{Tratamento}

0 tratamento recomendado das uretrites pode depender ou não de suporte laboratorial. ${ }^{2} \mathrm{Na}$ presença de queixa de corrimento uretral, após anamnese e exame físico, com corrimento uretral confirmado, caso não haja suporte de laboratório (uretrite sem identificação do agente etiológico), é indicado o tratamento de clamídia e gonorreia com azitromicina 1g, via oral (VO), dose única, e ceftriaxona 500mg, intramuscular (IM), dose única. Na possibilidade de realização de bacterioscopia (Gram), com visualização de diplococos intracelulares Gram-negativos, deve-se tratar gonorreia e clamídia conforme descrito acima (ceftriaxona $500 \mathrm{mg}$, IM, dose única, e azitromicina 1g, V0, dose única); a presença de diplococos intracelulares Gram-negativos indica infecção por gonococo, mas não exclui a possibilidade de infecção por clamídia. Na ausência desses achados, deve-se tratar apenas clamídia com azitromicina 1g, V0, dose única. Quando possível a realização de coleta de material biológico para cultura ou biologia molecular no laboratório, esta deverá ocorrer na primeira consulta. 0 tratamento deverá ser instituído de imediato e os resultados dos testes poderão ser analisados na consulta de retorno. ${ }^{2}$

Destaca-se que, a partir de resultados do Projeto SenGono 2015-2017 (descrito no subtópico Resistência aos antimicrobianos), a diretriz brasileira de tratamento recomenda nacionalmente a terapia dupla de ceftriaxona $500 \mathrm{mg}$, IM, dose única, associada à azitromicina 1g, V0, dose única, para a infecção gonocócica anogenital não complicada (uretra, colo do útero e reto). ${ }^{2}$

Após sete dias do tratamento, deve-se garantir uma consulta para reavaliação de sinais e sintomas e entrega de resultados de exames de cultura ou de biologia molecular, ou ambos, quando realizados.
A remissão da sintomatologia caracteriza cura. $\mathrm{Na}$ persistência de sintomas, é importante excluir reinfecção, realização de tratamento inadequado para clamídia e gonorreia, resistência antimicrobiana (principalmente relacionada ao gonococo e M. genitalium), trauma, irritação química ou inserção de corpos estranhos. Em complemento, devese tratar T. Vaginalis com metronidazol $250 \mathrm{mg}$, dois comprimidos, V0, duas vezes ao dia, por sete dias, e analisar os resultados dos exames para avaliar a necessidade de instituir novo tratamento conforme achado. Uma consulta para reavaliação deverá ser agendada para após sete dias, com instituição de alta na ausência de sinais e sintomas, e referenciamento para serviços especializados, na persistência desses. As opções de tratamento para uretrites estão apresentadas na Figura $3{ }^{2}$

\section{Vigilância, prevenção e controle}

As IST estão entre os problemas de saúde pública mais comuns no Brasil e no mundo. A atenção primária à saúde constitui o ponto de partida para atendimento às IST, com realização de ações de prevenção, diagnóstico, tratamento oportuno e adequado e encaminhamento dos casos que não competem a esse nível de atenção. Para possibilitar a integralidade do cuidado, é fundamental que os serviços se organizem para promover 0 acesso a outros níveis de atenção, quando necessário. ${ }^{30}$

A anamnese, a identificação das diferentes vulnerabilidades e o exame físico são importantes elementos na abordagem das pessoas com IST. Para garantir a qualidade do atendimento, a adesão ao tratamento e a retenção no serviço de saúde, o profissional de saúde necessita estabelecer uma relação de confiança com a pessoa com IST, assegurando a privacidade e confidencialidade das informações.

É importante que o profissional esteja disponível para diálogo e forneça informações sobre educação em saúde, abordando temas como agentes causais das IST, possíveis formas de transmissão, prevenção, diagnóstico e importância da adesão ao tratamento, além da definição de estratégia para seguimento, atenção às parcerias sexuais e acesso aos insumos de prevenção. ${ }^{31}$

A prevenção e controle das IST que causam uretrites compreendem o uso correto de preservativos durante as relações sexuais; rastreamento e testagem regular de 


\begin{tabular}{|c|c|c|c|}
\hline Condição clínica & Primeira opção & Segunda opção & Observações \\
\hline $\begin{array}{l}\text { Uretrite sem identificação do } \\
\text { agente etiológico }{ }^{a, b}\end{array}$ & $\begin{array}{l}\text { Ceftriaxona 500mg, intramuscular } \\
\text { (IM), dose única mais azitromicina } \\
500 \mathrm{mg}, 2 \text { comprimidos, via oral (V0), } \\
\text { dose única }\end{array}$ & $\begin{array}{l}\text { Ceftriaxona 500mg, IM, dose } \\
\text { única mais doxiciclina 100mg, } 1 \\
\text { comprimido, V0, 2x/dia, por } 7 \text { dias }\end{array}$ & \\
\hline $\begin{array}{l}\text { Uretrite gonocócica e demais } \\
\text { infecções gonocócicas NÃ } 0 \\
\text { complicadas (uretra, colo do útero, } \\
\text { reto e faringe) }{ }^{a, b}\end{array}$ & $\begin{array}{l}\text { Ceftriaxona 500mg, IM, dose } \\
\text { única mais azitromicina 500mg, } 2 \\
\text { comprimidos, V0, dose única }\end{array}$ & & \\
\hline Uretrite não gonocócicab & $\begin{array}{l}\text { Azitromicina } 500 \mathrm{mg}, 2 \text { comprimidos, } \\
\text { V0, dose única }\end{array}$ & $\begin{array}{l}\text { Doxiciclina } 100 \mathrm{mg}, 1 \text { comprimido, } \\
\text { V0, } 2 x / \text { dia, por } 7 \text { dias }\end{array}$ & $\begin{array}{l}\text { A resolução dos sintomas pode } \\
\text { levar até } 7 \text { dias após a conclusão } \\
\text { da terapia. }\end{array}$ \\
\hline Uretrite por clamídia ${ }^{b}$ & $\begin{array}{l}\text { Azitromicina } 500 \mathrm{mg}, 2 \text { comprimidos, } \\
\text { V0, dose única }\end{array}$ & $\begin{array}{l}\text { Doxiciclina } 100 \mathrm{mg}, 1 \text { comprimido, } \\
\text { V0, } 2 x / \text { dia, por } 7 \text { dias }\end{array}$ & $\begin{array}{l}\text { A resolução dos sintomas pode } \\
\text { levar até } 7 \text { dias após a conclusão } \\
\text { da terapia. }\end{array}$ \\
\hline $\begin{array}{l}\text { Retratamento de infecções } \\
\text { gonocócicas }\end{array}$ & $\begin{array}{l}\text { Ceftriaxona 500mg, IM, dose } \\
\text { única mais azitromicina 500mg, } 4 \\
\text { comprimidos, V0, dose única }\end{array}$ & $\begin{array}{l}\text { Gentamicina 240mg, IM } \\
\text { mais azitromicina 500mg, } 4 \\
\text { comprimidos, V0, dose única }\end{array}$ & $\begin{array}{l}\text { Para casos de falha de tratamento. } \\
\text { Possíveis reinfecções devem ser } \\
\text { tratadas com as doses habituais. }\end{array}$ \\
\hline $\begin{array}{l}\text { Uretrite por Mycoplasma } \\
\text { genitalium }^{b}\end{array}$ & $\begin{array}{l}\text { Azitromicina 500mg, } 2 \text { comprimidos, } \\
\text { V0, dose única }\end{array}$ & & \\
\hline Uretrite por Trichomonas vaginalis ${ }^{\mathrm{b}}$ & $\begin{array}{l}\text { Metronidazol } 250 \mathrm{mg}, 2 \text { comprimidos } \\
\text { V0, } 2 x / \text { dia, por } 7 \text { dias }\end{array}$ & $\begin{array}{l}\text { Clindamicina 300mg, V0, 2x/dia, } \\
\text { por } 7 \text { dias }\end{array}$ & \\
\hline $\begin{array}{l}\text { Infecção gonocócica disseminada } \\
\text { (exceto meningite e endocardite) }\end{array}$ & $\begin{array}{l}\text { Ceftriaxona } 1 \mathrm{~g} \text { IM ou IV ao dia, } \\
\text { completando ao menos } 7 \text { dias de } \\
\text { tratamento mais azitromicina } 500 \mathrm{mg} \text {, } \\
2 \text { comprimidos, V0, dose única }\end{array}$ & & \\
\hline Conjuntivite gonocócica no adulto & Ceftriaxona 1g, IM, dose única & & \\
\hline
\end{tabular}

Fonte: adaptado do Protocolo Clínico e Diretrizes Terapêuticas para Atenção Integral às Pessoas com Infecções Sexualmente Transmissíveis, 2020. ${ }^{13}$

Notas: a) Se houver alergia grave às cefalosporinas, indicar azitromicina $500 \mathrm{mg}$, quatro comprimidos, via oral, dose única (dose total: $2 \mathrm{~g}$ ); b) 0 s casos e as parcerias devem se abster de relações sexuais desprotegidas até que o tratamento de ambos esteja completo (ou seja, após o término do tratamento com múltiplas doses ou por sete dias após a terapia com dose única).

\section{Figura 3 - Tratamento de uretrites}

pessoas assintomáticas, realizada nas populações específicas; investigação e manejo dos casos sintomáticos; instituição de tratamento imediato, quando indicado; e tratamento de parcerias sexuais. 0 tratamento, além de ter função curativa, também visa à interrupção da cadeia de transmissão e à prevenção de complicações decorrentes das uretrites. ${ }^{2,31}$

0 rastreamento de gonococo e clamídia, em pessoas assintomáticas, está indicado nas seguintes situações: gestantes menores de 30 anos, na primeira consulta do pré-natal; pessoas vivendo com HIV, no momento do diagnóstico da infecção pelo HIV; pessoas com indicação de profilaxia pós-exposição ao HIV; pessoas com IST no momento do diagnóstico; pessoas que sofreram violência sexual, no primeiro atendimento e no atendimento quatro a seis semanas após; pessoas com prática sexual anal receptiva (passiva) sem uso de preservativo; e pessoas em uso de profilaxia pré-exposição ao HIV, semestralmente. ${ }^{2}$

Para que se interrompa a cadeia de transmissão das IST, é fundamental que os contatos sexuais das pessoas infectadas sejam tratados, mesmo quando assintomáticos. ${ }^{3}$ Portanto, essa informação deve ser repassada à pessoa com IST, ao tempo em que se fornecem instrumentos para comunicação e apoio até 0 final do atendimento. Deverá ser garantida a confidencialidade, ausência de coerção e proteção contra a discriminação. ${ }^{2}$

Em homens com sintomas de infecção por gonococo, C. trachomatis ou M. genitalium, todas as parcerias sexuais durante as oito semanas anteriores precisam ser examinadas e tratadas. Em casos assintomáticos, isso se aplica a todas as parcerias sexuais dos últimos seis meses. ${ }^{3}$ 


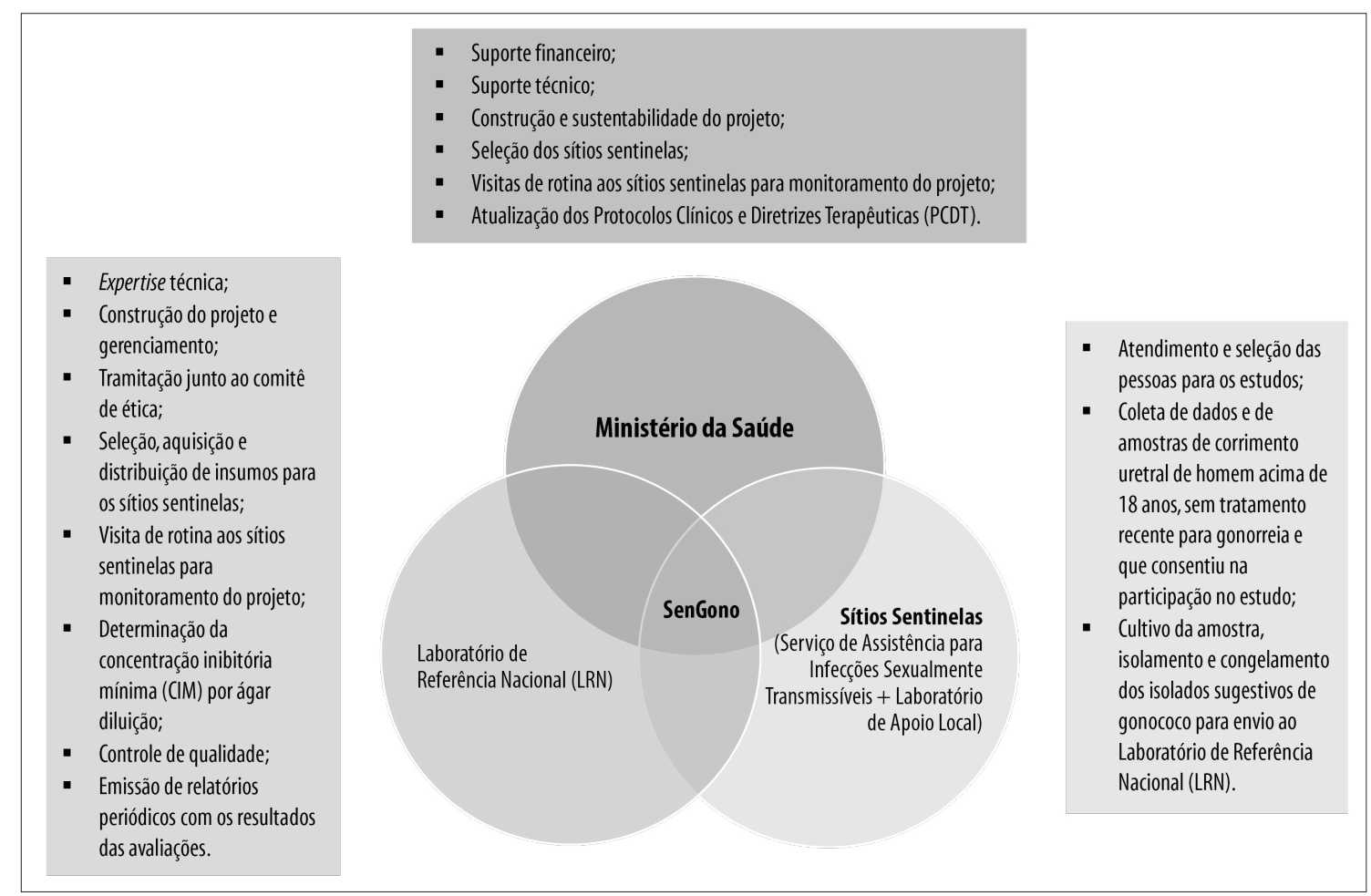

Fonte: adaptado do Boletim Epidemiológico nº 27/2020, do Ministério da Saúde. ${ }^{32}$

\section{Figura 4 - Funções do Ministério da Saúde, do Laboratório de Referência Nacional e dos Sítios Sentinelas no âmbito do Projeto SenGono}

As uretrites não são agravos de notificação compulsória nacional; entretanto, a síndrome do corrimento uretral é de notificação compulsória em alguns estados brasileiros. Ademais, o Ministério da Saúde do Brasil publicou uma portaria ${ }^{32}$ que instituiu sítios para vigilância sentinela da síndrome do corrimento uretral masculino, os quais devem fornecer informações para a construção de dados epidemiológicos oficiais. Além disso, visa-se fortalecer ações de prevenção de IST que causam corrimento uretral, subsidiar recomendações nacionais para o tratamento da síndrome, monitorar a susceptibilidade de gonococos aos antimicrobianos junto ao Projeto SenGono e pesquisar os agentes causais de úlceras genitais e a resistência de M. genitalium aos antimicrobianos. ${ }^{33}$

\section{Resistência aos antimicrobianos}

Ao longo da história da antibioticoterapia, a $N$. gonorrhoeae desenvolveu progressiva resistência aos antibióticos, desde as sulfonamidas até as fluorquinolonas. Após a disseminação da resistência ao ciprofloxacino, as cefalosporinas de terceira geração têm sido a base do tratamento, em associação à azitromicina. 0 surgimento de gonorreia resistente à cefalosporina impactará negativamente na capacidade dos profissionais para tratar a gonorreia de maneira eficaz. Cepas de gonococo consideradas multidroga-resistentes e extensivamente resistentes a medicamentos já são encontradas no continente americano, asiático, em vários países europeus e na Oceania. ${ }^{34-37}$ É, portanto, fundamental monitorar continuamente a resistência aos antibióticos e incentivar a pesquisa e o desenvolvimento de novos esquemas de tratamento. ${ }^{38,39}$

A OMS, no "Relatório Global de Vigilância às Infecções Sexualmente Transmitidas de 2018”, prioriza 0 controle das infecções gonocócicas pela possibilidade iminente de tornarem-se infecções não tratáveis. ${ }^{40,41}$ 0 Brasil é um dos países membros do programa para vigilância mundial da susceptibilidade do gonococo aos antimicrobianos (Gonococcal Antimicrobial Surveillance Programme, GASP)..$^{42}$ As atividades do 
GASP no Brasil acontecem no âmbito do Projeto SenGono, fruto de uma cooperação entre o Ministério da Saúde, o Laboratório de Referência Nacional (Laboratório de Biologia Molecular, Microbiologia e Sorologia da Universidade Federal de Santa Catarina) e os sítios sentinelas distribuídos por todas as regiões do país (Figura 4). ${ }^{33}$

Na primeira edição, ocorrida entre 2015 e 2017, constatou-se uma resistência elevada ao ciprofloxacino, com proporções próximas e até superiores a $50 \%$ em todas as regiões do país, e altas proporções de susceptibilidade às cefalosporinas de terceira geração (cefixima e ceftriaxona) ${ }^{26}$

A vigilância realizada no Projeto SenGono integrou 0 "Plano Nacional de Combate à Resistência aos Antimicrobianos 2019-2023”, e está na sua segunda edição (2018-2020), com a expansão para novos sítios sentinelas e avaliação da suscetibilidade do gonococo a dois novos antimicrobianos (espectinomicina e gentamicina). Além disso, incorporou-se a essa edição a determinação da etiologia do corrimento uretral masculino e a investigação da resistência de M. genitalium aos antimicrobianos. ${ }^{33}$

0 M. genitalium foi identificado pela primeira vez em 1980 e reconhecido como importante causa de uretrites não gonocócicas. ${ }^{25,43-45}$ Por não apresentar parede celular, antibióticos como os beta-lactâmicos (incluindo penicilinas e cefalosporinas) não são eficazes. ${ }^{29}$ A introdução da azitromicina, utilizada como terapia de dose única para infecções por clamídia, resultou na depuração do $M$. genitalium do trato urogenital, ocorrendo eliminação do patógeno sem 0 desenvolvimento de doença. ${ }^{25,43,46-50}$ Porém, estudos recentes indicam uma tendência ascendente na prevalência de infecções por $M$. genitalium resistentes aos macrolídeos (resistência transmitida) e casos de resistência induzida após terapia com azitromicina. ${ }^{3,43,50}$ Não há evidências de que um regime estendido com azitromicina $(1,5 \mathrm{~g})$ seja superior ao regime de $1 \mathrm{~g}$ em dose única. ${ }^{51} 0$ moxifloxacino permanece altamente ativo contra a maioria dos M. genitalium resistentes a macrolídeos. No entanto, foram publicados os primeiros casos clínicos de falha do tratamento com moxifloxacino. ${ }^{3,28,43,44,50-53}$ Portanto, o M. genitalium é um problema emergente, necessitando de uma vigilância frequente e de estudos com novas opções de diagnóstico e tratamento. ${ }^{27}$

0 aumento da resistência, nas últimas décadas, reforça a importância do diagnóstico etiológico e a escolha de tratamentos adequados. Em 2016, a Assembleia Mundial da Saúde adotou a "Estratégia do setor de saúde global sobre infecções sexualmente transmissíveis, 2016-2021" ${ }^{38}$ Essa estratégia inclui a rápida expansão de intervenções e serviços baseados em evidências para eliminar as IST como preocupação de saúde pública até 2030 .

\section{Contribuição dos autores}

De Lannoy LH, Gaspar PC, Da Silva RJC, Júnior EPN, De Oliveira EC contribuíram igualmente com a concepção, delineamento, redação e revisão crítica do manuscrito. Todos os autores aprovaram a versão final do trabalho e são responsáveis por todos os seus aspectos, incluindo a garantia de sua precisão e integridade.

\section{Agradecimento}

Os autores agradecem aos membros do grupo técnico de especialistas responsáveis pela elaboração do PCDT para Atenção Integral às Pessoas com IST em 2020 a contribuição substancial para a realização deste trabalho.

\section{Referências}

1. Brasil. Ministério da Saúde. Portaria MS/SCTIE $\mathrm{n}^{\circ} 42$, de 5 de outubro de 2018. Torna pública a decisão de aprovar o Protocolo Clínico e Diretrizes Terapêuticas para Atenção Integral às Pessoas com Infecções Sexualmente Transmissíveis (IST), no âmbito do Sistema Único de Saúde - SUS [Internet]. Diário Oficial da União, Brasília (DF), 2018 out 8 [citado 2020 jun 28];Seção 1:88. Disponível em: http://bvsms.saude.gov.br/bvs/ saudelegis/sctie/2018/prt0042_08_10_2018.html
2. Ministério da Saúde (BR). Secretaria de Vigilância em Saúde. Departamento de Doenças de Condições Crônicas e Infecções Sexualmente Transmissíveis. Protocolo clínico e diretrizes terapêuticas para atenção integral às pessoas com infecções sexualmente transmissíveis (IST) [Internet]. Brasília: Ministério da Saúde; 2020 [citado 2020 out 13]. 131p. Disponível em: http://www.aids.gov.br/pt-br/ pub/2015/protocolo-clinico-e-diretrizes-terapeuticas-para-atencao-integral-pessoas-com-infeccoes 
3. Buder S, Schöfer H, Meyer T, Bremer V, Kohl PK, Skaletz-Rorowski A, Brockmeyer N. Bacterial sexually transmitted infections. JDDG: J Deutsch Dermatol Gesellschaft [Internet]. 2019 Mar [cited 2020 0ct 13]; 17(3):287-315. Available from: https://doi.org/10.1111/ddg.13804

4. Rowley J, Vander Hoorn S, Korenromp E, Low N, Unemo M, Abud-Raddad L, et al. Chlamydia, gonorrhoea, trichomoniasis and syphilis: global prevalence and incidence estimates, 2016. Bull World Health Organ [Internet]. 2019 Aug [cited 2020 Jun 28]; 97(8):548-62. Available from: https://dx.doi.org/10.2471\%2FBLT.18.228486

5. Baumann L, Cina M, Egli-Gany D, Goutaki M, Halbeisen FS, Lohrer G, et al. Prevalence of Mycoplasma genitalium in different population groups: systematic review andmeta-analysis. Sex Transm Infect [Internet]. 2018 Jun [cited 2020 Jun 28]; 94(4):255-62. Available from: https://doi.org/10.1136/sextrans-2017-053384

6. European Centre for Disease Prevention and Control - ECDC. Gonorrhoea: annual epidemiological report for 2018 [Internet]. Stockholm: ECDC; 2019 [cited 2020 0ct 13]. 8 p. Available from: https:// www.ecdc.europa.eu/sites/default/files/documents/ gonorrhoea-annual-epidemiological-report-2018.pdf

7. Ministério da Saúde (BR). Secretaria de Ciência, Tecnologia e Insumos Estratégicos. Ceftriaxona $500 \mathrm{mg}$ para tratamento da Neisseria gonorrhoeae resistente à ciprofloxacina: relatório de recomendação n. 154 [Internet]. Brasília: Ministério da Saúde; 2015 [citado 2020 out 13]. 29 p. Disponível em: http://conitec.gov.br/images/Relatorios/2015/ Relatorio_Ceftriaxona_Gonorreia_final.pdf

8. Edwards JL, Apicella MA. The molecular mechanisms used by Neisseria gonorrhoeae to initiate infection differ between men and women. Clin Microbiol Rev [Internet]. 2004 0ct [cited 2020 Jun 20]; 17(4):965-81. Available from: https://doi.org/10.1128/cmr.17.4.965-981.2004

9. Fuchs W, Brockmeyer NH. Sexually transmitted infections. J Dtsch Dermatol [Internet].J Dtsch Dermatol Ges [Internet]. 2014 Jun [cited 2020 0ct 13]; 12(6):451-63. Available from: https://doi.org/10.1111/ddg.12310

10. Tu W, Ghosh P, Katz BP. A stochastic model for assessing chlamydia trachomatis transmission risk using longitudinal observational data. J R Stat Soc Ser A Stat Soc [Internet]. 2011 0ct [cited 2020
Oct 13]; 174(4):975-89. Available from: https:// doi.org/10.1111/j.1467-985x.2011.00691.x

11. Smieszek T, White PJ. Apparently-different clearance rates from cohort studies of mycoplasma genitalium are consistent after accounting for incidence of infection, recurrent infection, and study design. PLoS One [Internet]. 2016 Feb [cited 2020 0ct 13]; 11(2):e0149087. Available from: https://doi.org/10.1371/journal.pone.0149087

12. Thurman AR, Musatovova 0, Perdue S, Shain RN, Baseman JG, Baseman JB. Mycoplasma genitalium symptoms, concordance and treatment in high-risk sexual dyads. Int J STD AIDS [Internet]. 2010 Mar [cited 2020 0ct 13]; 21(3):177-83. Available from: https://doi.org/10.1258/ijsa.2009.008485

13. Walker J, Fairley CK, Bradshaw CS, Tabrizi SN, Chen MY, Twin J, et al. The difference in determinants of Chlamydia trachomatis and Mycoplasma genitalium in a sample of young Australian women. BMC Infect Dis [Internet]. 2011 [cited 2020 0ct 13]; 11:35. Available from: https://dx.doi.org/10.1186\%2F1471-2334-11-35

14. Francis SC, Kent CK, Klausner JD, Rauch L, Kohn R, Hardick A, et al. Prevalence of rectal Trichomonas vaginalis and Mycoplasma genitalium in male patients at the San Francisco STD clinic, 2005-2006. Sex Transm Dis [Internet]. 2008 Sep [cited 2020 Jul 10]:35(9):797-800. Available from: https:// dx.doi.org/10.1097\%2FOLQ.0b013e318177ec39

15. Penna G0, Hajjar LA, Braz TM. Gonorreia. Rev Soc Bras Med Trop [Internet]. 2000 out [citado 2020 jul 25]; 33(5):451-64. Disponível em: https:// doi.org/10.1590/S0037-86822000000500007

16. Gaydos C, Maldeis NE, Hardick A, Hardick J, Quinn TC. Mycoplasma genitalium compared to chlamydia, gonorrhoea and trichomonas as an aetiological agent of urethritis in men attending STD clinics. Sex Transm Infect [Internet]. 2009 Oct [cited 2020 Jul 25]; 85(6):438-40. Available from: https://doi.org/10.1136/sti.2008.035477

17. Daley GM, Russell DB, Tabrizi SN, McBride J. Mycoplasma genitalium: a review. Int J STD AIDS [Internet]. 2014 Jun [cited 2020 Jul 25]:25(7):475-87. Available from: https://doi.org/10.1177/0956462413515196

18. Lis R, Rowhani-Rahbar A, Manhart LE. Mycoplasma genitalium infection and female reproductive tract disease: a meta-analysis. Clin Infect Dis [Internet]. 2015 Aug [cited 2020 0ct 13]; 61(3):418-26. Available from: https://doi.org/10.1093/cid/civ312 
19. Brígido HAZ, Mourão JRM. Corrimentos uretrais. In: Leão RNQ (coord.), Bichara CNC, Fraiha NH, Vasconcelos PFC. Medicina tropical e infectologia da Amazônia. Belém: Samauma Editorial; 2013. p. 245-50.

20. Rimawi BH, Soper DE. Infectious diseases of the female reproductive and urinary tract. In: Hacker \& Moore's essentials of obstetrics and gynecology [Internet]. Philadelphia: Elsevier; 2016 [cited 2020 0ct 13]. p. 276-90. Disponível em: http://med-mu.com/wp-content/ uploads/2018/07/Hacker-Moore-s-Essentials-of-Obstetrics-and-Gynecology-6E-PDF-UnitedVRG-1-.pdf

21. Moherdaui F, Vuylsteke B, Siqueira LF, dos Santos Júnior MQ, Jardim ML, de Brito AM, et al. Validation of national algorithms for the diagnosis of sexually transmitted diseases in Brazil: results from a multicentre study. Sex Transm Infect [Internet]. 1998 Jun [cited 2020 Jun 5]; 74(Suppl 1):S38-43. Available from: https://pubmed.ncbi.nlm.nih.gov/10023352/.

22. Menezes Filho JR, Sardinha JCG, Galbán E, Saraceni $\mathrm{V}$, Talhari C. Effectiveness of syndromic management for male patients with urethral discharge symptoms in Amazonas, Brazil. An Bras Dermatol [Internet]. 2017 Nov-Dec [cited 2020 Jun 3]; 92(6):779-84. Available from: https://doi.org/10.1590/abd1806-4841.20175453

23. Wi TE, Ndowa FJ, Ferreyra C, Kelly-Cirino C, Taylor MM, Toskin I, et al. Diagnosing sexually transmitted infections in resource-constrained settings: challenges and ways forward. J Int AIDS Soc [Internet]. 2019 Aug [cited 2020 Jun 20]; 22(Suppl 6):e25343. Available from: https://doi.org/10.1002/jia2.25343.

24. World Health Organization - WHO. Laboratory diagnosis of sexually transmitted infections, including human immunodeficiency virus [Internet]. Geneva: World Health Organization; 2013 [cited 2020 Jun 20]. 228 p. Available from: https://apps.who.int/iris/ bitstream/handle/10665/85343/9789241505840_eng. pdf;jsessionid=89BC7AF948F36261070A5DB66F3CF2BA?sequence $=1$

25. Taylor-Robinson D. Diagnosis and antimicrobial treatment of Mycoplasma genitalium infection: sobering thoughts. Expert Rev Anti Infect Ther [Internet]. 2014 Jun [cited 2020 Oct 13]; 12(6):715-22. Available from: https://doi.org/10.1586/14787210.2014.919220

26. Bazzo ML, Golfetto L, Gaspar PC, Pires AF, Ramos MC, Franchini M, et al. First nationwide antimicrobial susceptibility surveillance for Neisseria gonorrhoeae in Brazil, 2015-16. J Antimicrob Chemother [Internet].
2018 Jul [cited 2020 Jun 12]; 73(7):1854-61. Available from: https://doi.org/10.1093/jac/dky090

27. Unemo M, Seifert HS, Hook EW, Hawkes S, Ndowa F, Dillon JR. Gonorrhoea. Nat Rev Dis Primers 5 [Internet]. 2019 Nov [cited 2020 Jun 15]; 5(1):79. Available from: https://doi.org/10.1038/s41572-019-0136-6

28. Murray GL, Bradshaw CS, Bissessor M, Danielewski J, Garland SM, Jensen JS, et al. Increasing macrolide and fluoroquinolone resistance in mycoplasma genitalium. Emerg Infect Dis [Internet]. 2017 May [cited 2020 0ct 13]; 23(5):809-12. Available from: https://dx.doi.org/10.3201\%2Feid2305.161745

29. Workowski KA, Bolan GA; Centers for Disease Control and Prevention - CDC. Sexually transmitted diseases treatment guidelines, 2015. MMWR Recomm Rep [Internet]. 2015 Jun [cited 2020 Jun 20]:64(RR-03):1-137. Available from: https://www. ncbi.nlm.nih.gov/pmc/articles/PMC5885289/.

30. Ministério da Saúde (BR). Secretaria de Atenção à Saúde. Departamento de Atenção Básica. Cadernos de atenção básica: HIV/Aids, hepatites e outras DST [Internet]. Brasília: Ministério da Saúde; 2006 [citado 2020 out 13]. 196 p. Disponível em: http:// bvsms.saude.gov.br/bvs/publicacoes/abcad18.pdf

31. Barberá, MJ, Serra-Pladevall J. Infección gonocócica: un problema aún sin resolver. Enferm Infecc Microbiol Clin [Internet]. 2019 Feb [cited 2020 Jun 3]; 37(7):458-66. Available from: https://doi.org/10.1016/j.eimc.2018.12.008

32. Brasil. Ministério da Saúde. Portaria MS/GM n ${ }^{0}$ 1.553, de 17 de junho de 2020. Altera a Portaria de Consolidação n ${ }^{0}$ 5/GM/MS, de 28 de setembro de 2017, para instituir a Vigilância Sentinela da Síndrome do Corrimento Uretral Masculino (VSCUM) [Internet]. Diário Oficial da União, Brasília (DF), 2020 jun 18 [citado 2020 out 13]; Seção 1:61. Disponível em: http://bvsms.saude.gov.br/bvs/ saudelegis/gm/2020/prt1553_18_06_2020.html

33. Ministério da Saúde (BR). Informe semanal sarampo - Brasil, semanas epidemiológicas 1 a 25, 2020. Bol Epidemiol [Internet]. 2020 Jul [citado 2020 out 13]; 51(27). Disponível em: https:// antigo.saude.gov.br/images/pdf/2020/July/09/ Boletim-epidemiologico-SVS-27-06.07.2020.pdf

34. Allen VG, Mitterni L, Seah C, Rebbapragada A, Martin IE, Lee C, et al. Neisseria gonorrhoeae treatment failure and susceptibility to cefixime 
in Toronto, Canada. JAMA [Internet]. 2013 Jan [cited 2020 Jun 3]:309(2):163-70. Available from: https://doi.org/10.1001/jama.2012.176575

35. Unemo M, Golparian D, Nicholas R, Ohnishi M, Gallay A, Sednaoui P. High-level cefixime- and ceftriaxone-resistant Neisseria gonorrhoeae in France: novel penA mosaic allele in a successful international clone causes treatment failure. Antimicrob Agents Chemother [Internet]. 2012 Mar [cited 2020 Jun 3]:56(3):1273-80. Available from: https://doi.org/10.1128/aac.05760-11

36. Unemo M, Nicholas RA. Emergence of multidrug-resistant, extensively drug-resistant and untreatable gonorrhea. Future Microbiol [Internet]. 2012 Dec [cited 2020 Jun 3]:7(12):1401-22. Available from: https://doi.org/10.2217/fmb.12.117

37. Lewis DA. Global resistance of Neisseria gonorrhoeae: when theory becomes reality. Curr Opin Infect Dis [Internet]. 2014 Feb [cited 2020 Jun 3]:27(1):62-7. Available from: https:// doi.org/10.1097/qco.0000000000000025

38. World Health Organization - WHO. Global health sector strategy on Sexually Transmitted Infections, 2016-2021 [Internet]. Geneva: World Health Organization; 2016 [cited 2020 Jun 10]. Available from: https://www.who. int/reproductivehealth/publications/rtis/ghss-stis/en/

39. Centers for Disease Control and Precention - CDC. Antibiotic resistance threats in the United States, 2019. Atlanta, GA: U.S. Department of Health and Human Services, CDC; 2019 [cited 2020 0ct 13]. Available from: www.cdc.gov/DrugResistance/Biggest-Threats.html

40. Tien V, Punjabi C, Holubar MK. Antimicrobial resistance in sexually transmitted infections. J Travel Med [Internet]. 2020 Feb [cited 2020 Jun 3]; 27(1):taz101. Available from: https://doi.org/10.1093/jtm/taz101

41. World Health Organization - WHO. Report on global sexually transmitted infection surveillance [Internet]. Geneva: World Health Organization; 2018 [cited 2020 Jun 7]. Available from: https://www.who.int/reproductivehealth/ publications/stis-surveillance-2018/en/

42. World Health Organization - WHO. The gonococcal antimicrobial surveillance programme (GASP) [Internet]. Geneva: World Health Organization; 2020 [cited 2020 Jun 15]. Available from: https://www.who.int/reproductivehealth/ topics/rtis/gonococcal_resistance/en/
43. Horner P, Blee K, Adams E. Time to manage Mycoplasma genitalium as an STI: but not with azithromycin 1g!. Curr Opin Infect Dis [Internet]. $2014 \mathrm{Feb}$ [cited 2020 0ct 13]; 27(1):68-74. Available from: https://doi.org/10.1097/qco.0000000000000030

44. Manhart LE, Jensen JS, Bradshaw CS, Golden MR, Martin DH. Efficacy of antimicrobial therapy for mycoplasma genitalium infections. Clin Infec Dis [Internet]. 2015 Dec [cited 2020 0ct 13]; 61(Suppl 8):S802-17. Available from: https://doi.org/10.1093/cid/civ785

45. Birger R, Saunders J, Estcourt C, Sutton AJ, Mercer $\mathrm{CH}$, Roberts T, et al. Should we screen for the sexually-transmitted infection Mycoplasma genitalium? Evidence synthesis using a transmission-dynamic model. Sci Rep [Internet]. 2017 Nov [cited 2020 Oct 13]; 7(1):16162. Available from: https:// dx.doi.org/10.1038\%2Fs41598-017-16302-8

46. Kikuchi M, Ito S, Yasuda M, Tsuchiya T, Hatazaki $\mathrm{K}$, Takanashi M, et al. Remarkable increase in fluoroquinolone-resistant Mycoplasma genitalium in Japan. J Antimicrob Chemother [Internet]. 2014 Sep [cited 2020 0ct 13]; 69(9):2376-82. Available from: https://doi.org/10.1093/jac/dku164

47. Salado-Rasmussen K, Jensen JS. Mycoplasma genitalium testing pattern and macrolide resistance: a Danish nationwide retrospective survey. Clin Infect Dis [Internet]. 2014 Jul [cited 2020 0ct 13]; 59(1):24-30. Available from: https://doi.org/10.1093/cid/ciu217

48. Jensen JS, Bradshaw C. Management of mycoplasma genitalium infections - can we hit a moving target? BMC Infect Dis [Internet]. 2015 Aug [cited 2020 0ct 13]; 15:343. Available from: https://doi.org/10.1186/s12879-015-1041-6.

49. Sethi S, Zaman K, Jain N. Mycoplasma genitalium infections: current treatment options and resistance issues. Infect Drug Resist [Internet]. 2017 Sep [cited 2020 0ct 13]; 10:283-92. Available from: https://dx.doi.org/10.2147\%2FIDR.S105469

50. Bissessor M, Tabrizi SN, Twin J, Abdo H, Fairley CK, Chen MY, et al. Macrolide resistance and azithromycin failure in a Mycoplasma genitalium-infected cohort and response of azithromycin failures to alternative antibiotic regimens. Clin Infect Dis [Internet]. 2015 Apr [cited 2020 0ct 13]; 60(8):1228-36. Available from: https://doi.org/10.1093/cid/ciu1162

51. Read TR, Fairley CK, Tabrizi SN, Bissessor M, Vodstrcil L, Chen MY, et al. Azithromycin 1.5g over 5 days compared to $1 \mathrm{~g}$ single dose in urethral 
mycoplasma genitalium: impact on treatment outcome and resistance. Clin Infect Dis [Internet]. 2017 Feb [cited 2020 0ct 13]; 64(3):250-6. Available from: https://doi.org/10.1093/cid/ciw719

52. Dionne-Odom J, Geisler WM, Aaron KJ, Waites KB, Westfall A0, Van Der Pol B, et.al. High prevalence of multidrug-resistant mycoplasma genitalium in human immunodeficiency virus-infected men who have sex with men in Alabama. Clin Infect Dis
[Internet]. 2018 Feb [cited 2020 0ct 13]; 66(5):796-8. Available from: https://doi.org/10.1093/cid/cix853

53. Gratrix J, Plitt S, Turnbull L, Smyczek P, Brandley J, Scarrott R, et al. Prevalence and antibiotic resistance of Mycoplasma genitalium among STI clinic attendees in western Canada: a cross-sectional analysis. BMJ Open [Internet]. $2017 \mathrm{Jul}$ [cited 2020 0ct 13]; 7(7):e016300. Available from: https://doi.org/10.1136/bmjopen-2017-016300

\section{Abstract}

This article approach infections that cause urethral discharge, theme which is part of the Clinical Protocol and Therapeutic Guidelines for Comprehensive Care for People with Sexually Transmitted Infections, published by the Ministry of Health of Brazil in 2020. These guidelines were prepared based on scientific evidence and validated in discussions with experts. When urethritis is not treated correctly, or when the microorganism develops antimicrobial resistance, it can cause serious and even irreversible health damage. It is noteworthy that the high levels of antimicrobial resistance developed by pathogens that causes urethritis comprises a global emergency in public health. This article presents epidemiological and clinical aspects, recommendations on diagnostic and treatment, and strategies for surveillance, prevention and control actions of infections that cause urethral discharge, with the purpose of contributing with managers and health professionals to care qualification.

Keywords: Urethritis; Neisseria gonorrboeae; Chlamydia trachomatis; Clinical Protocols; Public Health.

\section{Resumen}

El artículo trata de las infecciones que causan secreción uretral, tema que hace parte del Protocolo Clínico y Directrices Terapéuticas para Atención Integral a Personas con Infecciones de Transmisión Sexual, publicado por el Ministerio de Salud de Brasil en 2020. Dicho documento se elaboró con base en evidencias científicas y se validó en discusiones con expertos. Las uretritis, cuando no tratadas correctamente o cuando el microorganismo desarrolla resistencia al tratamiento, puede ocasionar daños graves a la salud. Los niveles de resistencia antimicrobiana que estos agentes desenvuelven son considerados una emergencia de salud pública. En este artículo, se presentan aspectos epidemiológicos y clínicos, recomendaciones para el diagnóstico y tratamiento y estrategias para acciones de monitoreo epidemiológico, prevención y control de las infecciones que causan secreción uretral, a fin de contribuir con gerentes y personal de salud para la calificación de la asistencia.

Palabras clave: Uretritis; Neisseria gonorrboeae; Chlamydia trachomatis; Protocolos Clínicos; Salud Pública.

Recebido em 31/07/2020

Aprovado em 01/10/2020 


\section{Errata}

No artigo "Protocolo Brasileiro para Infecções Sexualmente Transmissíveis 2020: infecções que causam corrimento uretral", doi: 10.1590/\$1679-4974202100009.esp1, publicado no periódico Epidemiologia e Serviços de Saúde, v. 30(Esp.1):1-13, nas páginas 7 e 9:

Onde se lia:

Figura 3 - Tratamento e seguimento de casos de sífilis e neurossífilis

Leia-se:

Figura 3 - Tratamento de uretrites

Onde se lia:

A moxifloxacina permanece altamente ativa contra a maioria dos $M$. genitalium resistentes a macrolídeos.

No entanto, foram publicados os primeiros casos clínicos de falha do tratamento com moxifloxacina.

Leia-se:

0 moxifloxacino permanece altamente ativo contra a maioria dos $M$. genitalium resistentes a macrolídeos.

No entanto, foram publicados os primeiros casos clínicos de falha do tratamento com moxifloxacino. 